\title{
Structure and dynamics of cylindrical micelles at equilibrium and under shear flow
}

\author{
C.-C. Huang, ${ }^{1}$ J.-P. Ryckaert, ${ }^{2}$ and H. $\mathrm{Xu}^{3}$ \\ ${ }^{1}$ Institut für Festkörperforschung, Forschungszentrum Jülich GmbH, 52425 Jülich, Germany \\ ${ }^{2}$ Physique des Polymères, Université Libre de Bruxelles, Campus Plaine, CP 223, B-1050 Brussels, Belgium \\ ${ }^{3}$ Laboratoire de Physique des Milieux Denses, Université Paul Verlaine-Metz, 1 Boulevard Arago, 57078 Metz Cedex 3, France
}

(Received 28 November 2008; published 9 April 2009)

\begin{abstract}
The dynamics and rheology of semidilute unentangled micellar solutions are investigated by Langevin dynamics mesoscopic simulations coupled to a microreversible kinetic model for scissions and recombinations. Two equilibrium state points, differing by the scission energy and therefore by the corresponding average micelle length, have been examined. The kinetic rates are tuned by an independent parameter of the model, whose range is chosen in such a way that the kinetics always strongly couple to the chain dynamics. Our results confirm, as predicted by Faivre and Gardissat, that the stress relaxation, as well as the monomer diffusion, is characterized by a time $\tau_{\Lambda}$, defined by the lifetime of a segment $\Lambda$, whose Rouse relaxation time is equal to its lifetime. Moreover, the power-law dependence of the zero-shear viscosity versus $\tau_{\Lambda}$ was evidenced. Under stationary shear, the chains are deformed and their average bond length is increased, which enhances the overall scission frequency. In turn, this induces an overall shortening of the chains in order to increase the overall corresponding chain-end recombination frequency, as required by the stationary conditions. Nonequilibrium simulations show that the chain deformation and orientation, as well as the rheology of the system, can be expressed as universal functions of a single reduced shear rate $\beta_{\Lambda}=\dot{\gamma} \tau_{\Lambda}$ (with $\dot{\gamma}$ the bare shear rate). Furthermore, local analysis of the kinetics under stationary shear gives insights on the variation of the average length with shear rate.
\end{abstract}

DOI: 10.1103/PhysRevE.79.041501

PACS number(s): 83.80. Qr, 83.60.Rs, 82.20.Wt

\section{INTRODUCTION}

Cylindric micellar solutions, which belong to the broader family of the supramolecular polymers, have attracted much attention for the past 20 years [1-3]. Several recent books and review articles [3-5] present the state of the art of theoretical and experimental investigations on such systems, focusing mainly on their rheological and scattering properties. The reversible dynamics of micelles aggregation (by end-toend recombination) and disaggregation (by scission into two parts) give to these solutions peculiar viscoelastic properties exploited by the oil industry [6]. Numerous laboratory experiments have been performed to probe the linear and nonlinear viscoelasticity of cylindric micellar solutions leading, particularly in the nonlinear regime, to intriguing phenomena such as shear banding [7] and shear thickening [8], phenomena that also motivated intense theoretical investigations $[3,9,10]$.

The first "microscopic" theoretical study of the dynamics of micellar solutions was dealing with their linear viscoelastic behavior in the entangled regime [11]. Micelles were treated as ideal equilibrium polymers, characterized by an exponential distribution of lengths $P(L) \propto \exp \left(-L / L_{0}\right)$ with mean $L_{0}$. The classical polymer melts dynamics [12] was adapted to account for the scission-recombination kinetics, which introduces an extra stress relaxation mechanism. Using the mean-field (MF) approach of the kinetics, it is assumed that a chain end created by scission (through a unimolecular process with rate $k_{s}$ per unit length) will recombine with the chain end of another chain (through a bimolecular process with rate $k_{r}$ ). As a result of detailed balance requiring that $\frac{k_{s}}{k_{r}}=\frac{\phi}{2 L_{0}^{2}}$, where $\phi$ is the monomer number density (or volume fraction), the time $\tau_{b}=\left(k_{s} L_{0}\right)^{-1}$ character- izing the kinetics is the average lifetime of a micelle of average length and also the lifetime of a chain end. Applying a small step shear strain at $t=0$, the resulting shear stress can be expressed as $G(t)=G_{0} u(t)$, where $G_{0}$ is the elastic shear modulus and $u(t)$ is a normalized relaxation function that decays to zero on the structural relaxation time $\tau_{\text {relax }}$ of the system. The zero-shear rate viscosity is then expressed as $\eta_{0}=G_{0} \tau_{\text {relax }}$. For a monodisperse entangled melt of usual polymers of length $L, \tau_{\text {relax }}=\tau_{\text {rep }}(L)$, where $\tau_{\text {rep }}(L)$ is the reptation time, while for an entangled micellar solution where $\tau_{b} \leqslant \tau_{\text {rep }}\left(L_{0}\right)$, one expects $\tau_{\text {relax }} \leqslant \tau_{\text {rep }}\left(L_{0}\right)$.

The viscoelastic response of entangled systems is generally obtained by identifying the function $u(t)$ with the tube, relaxation function. In standard polymer dynamics theory, the tube relaxation results only from the reptation motion of the chain out of its original tube, while for micelles with sufficiently rapid kinetics, the chain scissions introduce locally an additional partial relaxation of the tube. The way to express this local relaxation depends on the nature of the dynamics of the new chain ends on the $\tau_{b}$ time scale and on the ratio of the breaking and reptation times $\zeta=\frac{\tau_{b}}{\tau_{\text {rep }}}$. In the regime $N_{T}^{-1}<\zeta<1$, where $N_{T}$ is the number of tube segments, Cates [3] obtained $\tau_{\text {relax }}=\sqrt{\tau_{b} \tau_{\text {rep }}\left(L_{0}\right)}$, the relaxation being exponential only if the kinetics is sufficiently fast, namely $\zeta^{1 / 2}<1$. If the latter inequality is not verified strongly enough, nonexponential corrections appear in the relaxation function as the local stress relaxation is not uniform along the tube. If the kinetics is even faster such that $\zeta<N_{T}^{-1}$, different $\tau_{\text {relax }}$ expressions are obtained depending on whether the chain end dynamics on the $\tau_{b}$ time scale is simply Rouse-like [13] or in the "breathing modes" regime [3].

The mean-field hypothesis for the scission-recombination kinetics has been challenged by [14] in the context of en- 
tangled micelles in the regime $N_{T}^{-1}<\zeta<1$ where the end chain dynamics is diffusive along the tube contour on the $\tau_{b}$ time scale. The nonexponential contributions to the shear stress relaxation observed in some experimental rheological data in the short-time regime were attributed to successive correlated scission-recombination events whereby the two chain ends produced by scission recombine with each other if $\tau_{b}<\tau_{h}\left[\tau_{h}\right.$ is the time needed by a chain end to diffuse over a distance $h=\left(\frac{L_{0}}{\phi}\right)^{1 / 3}$ corresponding to the typical distance between chain ends in a micellar solution of number density $\phi]$. A key parameter is thus $X=\frac{\tau_{h}}{\tau_{b}}$ : if $X \ll 1$, the micellar dynamics is "mean-field"-like, while in the opposite case $(X \gg 1)$, the dynamics is coined "diffusion-controlled" (DC) and leads to qualitatively different results. The distribution of recombination times $\psi(t)$ is no longer a single exponential as for $\mathrm{MF}$ cases but presents two successive regimes. For times below $\tau_{h}$, it presents an algebraic decay $\psi(t)$ $\approx\left(t^{*}\right)^{-1}\left(t^{*} / t\right)^{5 / 4}$, where $t^{*}$ is the time for two newly created chain ends to recombine with one another $\left(t^{*}<\tau_{h}\right.$ in the DC case). For $t>\tau_{h}$, the distribution becomes exponential, $\psi(t)$ $\approx \frac{\tau_{b}}{\tau_{h}^{2}} \exp \left(-t / \tau_{h}\right)$. The shear relaxation function thus also presents two different regimes where, at long times $\left(t>\tau_{h}\right)$, one gets an exponential decay with a characteristic time $\tau_{\text {relax }}$ equal to $\sqrt{\tau_{h} \tau_{\text {rep }}\left(L_{0}\right)}$, in which the breaking time of the MF expression is replaced by an effective time equal to $\tau_{h}$, assumed to be the typical minimum time needed for a chain end to make a noncorrelated recombination with a new partner. A very recent refined theoretical analysis of the diffusion-controlled dynamics of entangled micelles [15] has further shown that other regimes exist where the stress relaxation can be highly nonexponential at long times. It should be noted that in all the above theoretical approaches that are valid for entangled solutions, the main relaxation time always depends on the micelle average size $L_{0}$, whatever the time scale over which the kinetics proceeds.

For unentangled micellar solutions, it is expected that, if the scission-recombination mechanism is fast enough, the structural relaxation will be characterized by $\tau_{\text {relax }}<\tau_{R}\left(L_{0}\right)$, where $\tau_{R}\left(L_{0}\right)=\tau_{0} L_{0}^{2}$ is the Rouse relaxation time of a chain of size $L_{0}$ in an equivalent polymer melt, with $\tau_{0}$ a local time. To deal with this new situation, Faivre and Gardissat proposed a modification of the standard Rouse theory for stress relaxation [16] within the context of the viscoelasticity of liquid selenium where temporary linear atomic structures, akin to cylindric micelles, are present. Within the spirit of mean-field kinetics, they took into account the effects of independent scissions taking place along the chains while they implicitly assumed that the recombination events remain neutral on the relaxation process. In the standard theory of unentangled polymer dynamics, the stress relaxation takes the form of a sum of exponential terms associated with the different Rouse modes, a particular Rouse mode representing the collective dynamics of a set of $n$ adjacent "monomers." If a subsegment of $n$ monomers is the object of random scissions, the lifetime distribution is exponential with a characteristic time $\tau_{n}=\left(n k_{s}\right)^{-1}$. Hence, these scissions are supposed to cause the amplitude of the associated mode relaxation, a constant in the standard Rouse expression, to decay exponentially in time with a characteristic time equal to the $n$-monomer segment average lifetime $\tau_{n}$. By summation over all modes, the final expression for the fast kinetics case is $G(t) \propto \frac{1}{\sqrt{t}} \exp \left(-\frac{t}{\tau}\right)$, where the relevant time scale $\tau$ $=\tau_{b}^{2 / 3} \tau_{R}\left(L_{0}\right)^{1 / 3}$ is now independent of $L_{0}$. The viscoelastic behavior of unentangled micelles subject to fast scissionrecombination kinetics is thus found to be related to internal chain segment dynamical effects, the static average length of the micelles $\left(L_{0}\right)$ in the solution becoming irrelevant.

The above summary indicates that, already in the linear regime, the cylindric micelles solutions present a rich variety of rheological behaviors depending upon the relative importance of a few physically relevant time scales. Experimentally, Cole-Cole plots on the linear viscoelasticity of entangled micelles have shown, for a wide range of different chemical types, a strongly Maxwellian character. The reptation-reaction model [3] has proved to be successful not only to justify the exponential character of the relaxation but also to interpret the origin of leading corrections to this behavior observed experimentally in some particular cases. By contrast, the case of unentangled melts has been much less investigated experimentally [16].

Further investigations focusing on the dynamics of micellar solutions were performed by molecular dynamics or Brownian dynamics simulations [17-22]. These simulation studies either use existing polymers generic models suitably modified to allow for reversible scissions and recombinations, or they explore new models within a multiscale strategy to approach more realistically giant micelles rheology [23]. Actually, a wide literature exists today on micellar solutions simulations using either lattice models or continuous models, but the focus is often put on structural properties, in particular the micelle length distribution, at various concentrations or temperatures, at equilibrium or under shear flow conditions. In shear flow, it has been quite generally observed $[17,19,24,25]$ that the mean size of the micelles decreases with increasing shear flow. Studies where the focus is directed toward the coupling between polymer dynamics and the explicit scission-recombination kinetics, and its rheological implications, were addressed by much fewer simulation studies.

Using the FENE-C model [17], which is a continuous bounding pair potential with a barrier $E_{\mathrm{sc}}$ to scission but presenting no barrier to recombination, Padding and Boek [22] simulated by molecular dynamics a dense fluid of monomers at high (liquid) monomer volume fraction. For various values of $E_{\mathrm{sc}}$ (which also represents the energy gain when two micelles fuse together), they simulated micelles of various average lengths $4<L_{0}<140$. By looking to the explicit distribution of chain end recombination times $\psi(t)$, they observed two main successive time regimes, in very close analogy to the situation conjectured by O'Shaughnessy and $\mathrm{Yu}$ in the context of entangled melts with correlated transitions [14]. From an analysis of the simulation data, they got a $\left(t / t^{*}\right)^{-5 / 4}$ decay at short times, followed by an exponential behavior at long times. As they noted, the first algebraic part can be explained by the theoretical argument used by O'Shaughnessy et al. [14], as the mean squared displacement of chain ends in the present unentangled case follows a $t^{1 / 2}$ power law, just like it does in the reptation regime for an entangled melt where one-dimensional curvilinear diffusion 
takes place along the "random walk" tube. The presence of correlated scission recombination events between the same partners in MD simulations is indeed highly plausible, given the form of the pair interaction, which easily allows two chain ends generated a short time earlier by scission to recombine with each other after some Brownian motion, especially in the absence of any energy barrier to their recombination. The authors interpreted the time scale of the final exponential behavior as the average time $t_{h}$, which is the time for a chain end produced by scission to reach a new chain end. They observed that the stress relaxation function computed from the MD trajectory was compatible with a Faivre-Gardissat expression with relaxation time expressed as $\tau=\tau_{h}^{2 / 3} \tau_{R}\left(L_{0}\right)^{1 / 3}$, where $\tau_{h}$ was set to the value extracted from the long-time behavior of $\psi(t)$. The same authors and Briels [25] studied very recently the rheology of micelles on the basis of a new model based on articulated rods to better cope with the micelle persistence length, allowing for scissions and recombinations through the use of a continuous potential with a barrier separating unbounded and bounded states.

Unentangled micellar solutions were also studied by Brownian or Langevin dynamics on the basis of a cylindric micelle model built as a linear assembly of Brownian particles where scissions and recombinations are modeled by a stochastic process interchanging the nature (bounded or unbounded) of the two-body potential between neighboring particles $[19,20,26]$. Each monomer possesses two arms that can be free (such as in isolated monomer or in chain end monomer) or engaged in a bounding pair with one arm of another monomer, a model allowing linear micellar structures only. A Lennard-Jones-FENE (LJF) restraining potential is used for binding two arms while a purely repulsive LJ potential is considered for unbound pairs. The bounded potential is shifted vertically so that its minimum lies at an energy $W$ below the "zero potential level" of the unbounded form (large distance value), representing a decrease or an increase of energy when a bond forms or breaks. The scission-recombination kinetics is controlled by an adjustable control parameter, the rate $\omega$ at which arms are selected at random and a change of their status attempted [26,20].

This model offers two important control parameters: for a given $(\phi, T)$ state point, $W$ fixes the mean micelle length $L_{0}$ while $\omega$ controls the size of the barrier heights and thus the scission-recombination rates. The scission rate can be expressed as $k_{s}=2 \omega Q_{s}$, where $Q_{s}$ is the probability that a bond opening is successful, once one of the arms engaged in the bond has been selected. As at equilibrium $Q_{s}$ is a product of the imposed frequency $W$ and the probability $Q_{s}$, as a function of the state point $T, \phi W$, while thermodynamic properties are independent of $\omega$, our kinetics model allows us to investigate the effect of the sole modification of the internal kinetics time scale. Our earlier studies on a single state point (system of 1000 beads at $\phi=0.15, k_{B} T=1$, and $W=10 k_{B} T$ ) for micelles of average size $L_{0}=56$ led to a distribution of first recombination times having the mathematical features observed by Padding and Boek (algebraic decay at short times followed by exponential decay with characteristic time, which we denoted as $\tau_{b}$ ). We observed that all dynamical results are consistent with theoretical predictions based on a MF approach of the kinetics [19,20], provided the rates are renormalized as $k_{s}=2 \omega \kappa Q_{s}$ and $\tau_{b}=\left(2 \omega \kappa Q_{s} L_{0}\right)^{-1}$, in which the renormalizing factor $0<\kappa(\omega)<1$ is such that $(1-\kappa)$ measures the fraction of correlated scissionrecombination at short times, at a given state point. In simulations of micellar solutions with a microscopic model allowing scission-recombinations, it is crucial to distinguish between the bare rates (e.g., $k_{s}^{0}=2 \omega Q_{s}$ ) and the effective rates (e.g., $k_{s}=\kappa k_{s}^{0}$ ) where only the effective ones matter in the "long-time" window of interest for unentangled solutions. The relevance of these effective rates was demonstrated in simulated "T-jump experiments" $[18,20]$ and in the interpretation of the shear stress relaxation (or viscosity) according to the Faivre-Gardissat expression where the relaxation time $\tau=\tau_{b}^{2 / 3} \tau_{R}\left(L_{0}\right)^{1 / 3}$ implies the lifetime $\tau_{b}$ based on effective rates $[19,20]$. While these observations are coherent with earlier results [22], it appears impossible however in the explored parameter range of our model to relate the $\omega$-dependent effective lifetime $\tau_{b}$ to the $\omega$-independent chain end diffusion time $\tau_{h}$.

In the present paper, we further investigate dynamical properties of our model by Langevin dynamics. In particular, equilibrium dynamics and rheological properties of our system are explored through two state points differing by the "end cap" energy parameter $W$, leading to average micelle lengths of $L_{0}=56$ and 151. The paper is organized as follows. In Sec. II, we review the main features of the model and its implementation. In Sec. III, we establish the microscopic expressions of the rate constants, and we test explicitly that our Langevin dynamics plus the scission-recombination stochastic procedure satisfies microscopic reversibility. In Sec. IV, we discuss the link between the effective rate constants and the transport properties of the system. Section V is devoted to the properties of our system under stationary shear flow. Section VI gives some conclusions of the present study.

\section{MODEL AND SIMULATION TECHNIQUE}

The micelles are modeled as flexible equilibrium polymer (EP) chains composed of spherical beads of mass $m$ interacting via pairwise interactions shown in Fig. 1. Nonbounded pairs are interacting via a repulsive Lennard-Jones $U_{2}(r)$ given by Eq. (1). Chains are linear sequences of $n$ beads directly connected by a set of $(n-1)$ bounding potentials $U_{1}(r)$ of the Lennard-Jones-FENE type [27], given in Eq. (2). It is important to stress that the bounding potential is shifted vertically so that its minimum lies at an energy value $-W$ with respect to the (zero) energy level of the repulsive LJ potential $U_{2}(r)$ at large distance. We have

$$
\begin{gathered}
U_{2}(r)=4 \epsilon\left[\left(\frac{\sigma}{r}\right)^{12}-\left(\frac{\sigma}{r}\right)^{6}+\frac{1}{4}\right] \Theta\left(2^{1 / 6} \sigma-r\right), \\
U_{1}(r)=U_{2}(r)-0.5 k R^{2} \ln \left(1-\left[\frac{r}{R}\right]^{2}\right)-U_{\min }-W,
\end{gathered}
$$

where $\Theta(x)$ is the Heaviside function $[\Theta(x)=0$ or 1 for $x$ $<0$ or $x \geqslant 0], U_{\min }$ is the minimum of the sum of the first two terms in $U_{1}, R$ is the maximum extension of the bond, and $k$ 


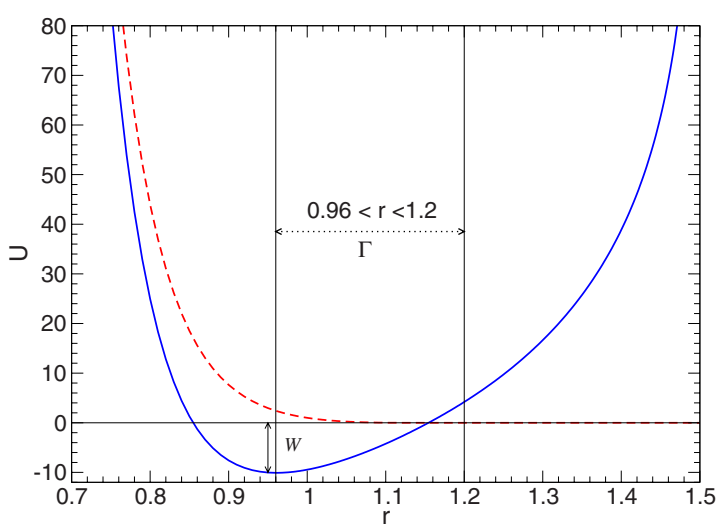

FIG. 1. (Color online) Bounded potential $U_{1}(r)$ (continuous curve) and unbounded potential $U_{2}(r)$ (dashed line) between a pair of monomers. $W$ is a parameter tuning the energy required to open the bond. The figure also shows the $\Gamma$ region where potential swaps (equivalent to bond scissions or bond recombinations) are allowed. The energy is scaled by $\epsilon$ and the length by $\sigma$. This figure corresponds to $W=10, R=1.5$, and $k=30$. The two last parameters and the $\Gamma$ region are fixed through the whole study.

is a spring constant. As potential swaps $U_{1}(r) \rightleftharpoons U_{2}(r)$ are considered to model scissions or recombinations, $W$ thus represents the pair bounding energy or, in the present context, twice the end cap energy in a cylindric micelle.

We study a system of $M$ beads in a volume $V$, in a solvent of temperature $T$, modeled as a continuum with friction coefficient $\xi$. The space-time evolution of the beads is governed by a Langevin dynamics (LD) scheme. The equation of motion of the bead " $i$ " is

$$
m \frac{d^{2} \bar{r}_{i}}{d t^{2}}=\bar{F}_{i}-\xi\left[\bar{v}_{i}-\bar{v}^{0}\left(\bar{r}_{i}\right)\right]+\bar{R}_{i}
$$

where $\bar{r}_{i}, \bar{v}_{i}$, and $\bar{F}_{i}$ are, respectively, the position, velocity, and systematic force of bead $i$, and $\vec{v}^{0}(\vec{r})$ is the macroscopic flow field term, which is absent in equilibrium simulations. In the presence of shear flow, we adopt the Lees-Edwards boundary conditions [28]. The above equations neglect the hydrodynamic interactions, as in some previous works on complex fluids $[29,30]$. The random force $\bar{R}_{i}$ obeys the usual Langevin dynamics hypothesis and fluctuation-dissipation theorem [31], i.e., $\left\langle R_{i \alpha}(t) R_{j \beta}\left(t^{\prime}\right)\right\rangle=2 m \xi k_{B} T \delta_{i j} \delta_{\alpha \beta} \delta\left(t-t^{\prime}\right)$, where the Greek-letter subscripts represent Cartesian components. The flow field $\bar{v}^{0}(\bar{r})$ is $\bar{v}^{0}(\bar{r})=\dot{\gamma} y \overline{1}_{x}$ for the system under shear flow along $x$ and velocity gradient $\dot{\gamma}$ along $y$.

In our model, each bead possesses two arms that can be either in a bounded or an unbounded state (branching and cyclic chains are excluded). A key control parameter is $\omega$, the scission-recombination trial frequency per arm. After each LD time step $\Delta t$, an attempt to modify the status of each arm is made with a probability $\omega \Delta t$. Such an attempt also involves the change of status of a second arm, namely the partner arm in the breaking of an existing bond or the complementary free arm needed in the forming of a new bond. Any attempt thus merely consists in going from a topological "state" to another topological "state" (with identi- cal bead positions), which only differ by the presence or absence of a particular bond. The full trial-acceptance procedure involving the original arm follows a microreversible METROPOLIS Monte Carlo (MC) algorithm, which has been explained in detail in Ref. [26], for which we establish below a link with kinetic rates.

All experiments are performed under the conditions of temperature $T=1$, monomer density $\phi=0.15$, and friction coefficient $\xi=3 \pi$, where all quantities are reduced by appropriate combinations of the bead mass $m$, the length $\sigma$, and energy $\epsilon$ appearing in the Lennard-Jones potential $U_{2}(r)$. Equations (3) are solved by the algorithm suggested in Ref. [32], using a time step $\Delta t=0.005$. We studied two semidilute thermodynamic state points differing only by the choice of the bond scission energy parameter $W$. For the case $W=10$, we used $M=1000$ beads and we get an average length $L_{0}$ $=56$ at equilibrium. For $W=12$, we get longer chains with $L_{0}=151$ at equilibrium and we used $M=5000$ beads. In both cases, the average chain size indicates semidilute unentangled conditions for our system, namely $L^{*}<L_{0}<L_{e}$. Indeed, $L_{0}$ is larger than the threshold length $L^{*}$ between the dilute and the semidilute regimes given by $L^{*}=\phi^{1 /(1-3 \nu)}$ $\approx 13$, where $\nu$ is the Flory exponent. Our systems are unentangled as $L_{0}$ is much smaller than the entanglement length $L_{e} \approx 630$ for our system, using the scaling $L_{e}(f)$ $=L_{e}(1) p^{-1 /(3 \nu-1)}$ (see Ref. [33], where $p$ is the packing fraction, and the estimate $L_{e}(1)=65$ for a melt of FENE chains at temperature $T=1$ at a packing fraction that is larger by a factor $\left(\frac{0,85}{0,15}\right)$, see Ref. [34]).

The rate $\omega$ was varied in the range $0.02 \leqslant \omega \leqslant 5$ as it turned out to be the adequate window for this control parameter to be in a regime where the resulting scissionrecombination kinetics is sufficiently fast to affect the structural relaxation time of our micellar solutions. Table I gives the list of all equilibrium simulations performed, including the total duration $T_{s}$ of the runs.

\section{SCISSION AND RECOMBINATION EVENTS AT EQUILIBRIUM AND RELATED KINETIC CONSTANTS}

\section{A. Microscopic reversibility and "bare" kinetic constants}

In this section, we will express the scission and recombination rate constants in microscopic terms, hence clarifying the nature of our kinetics algorithm, which is based on two successive steps: selecting an arm at random (with frequency $\omega)$ and then looking for the possibility to switch the status, which can be either free or bounded, of that arm. By analyzing how individual scission and recombination events are distributed in terms of the relative distance between involved monomers, a detailed check of the microscopic reversibility of the stochastic process is allowed.

We start by relating the total number of successful MonteCarlo scission or recombination steps to the bare scission and recombination rate constants $k_{s}^{0}$ and $k_{r}^{0}$, the terminology of "bare rates" being needed to distinguish them from "effective rates" that we introduce later, dealing with long-time relaxation. For an equilibrium state sampled with our algorithm, 
TABLE I. Static and kinetic data for the two semidilute cases, namely $W=10\left(L_{0}=56\right)$ and $W=12\left(L_{0}=151\right)$, for the various $\omega$ values investigated. $T_{s}$ is the total simulation time exploited for statistics. $Q_{s}^{\mathrm{eq}}$ and $Q_{r}^{\mathrm{eq}}$ are MC scission and recombination probabilities, defined in the text, which enter in the microscopic expressions of the rate constants. $\kappa$ gives the fraction of transitions that are effective in the determination of long-time scission and recombination rate constants $k_{s}$ and $k_{r}$. $\tau_{b}=\left(k_{s} L_{0}\right)^{-1}$ is the average effective lifetime of a chain end or equivalently the average effective lifetime of a chain of length $L_{0}$. Finally, the quantity $f^{e}$ gives the fraction of recombinations of a free arm with a new free arm partner.

\begin{tabular}{|c|c|c|c|c|c|c|c|c|c|}
\hline W & $\omega$ & $T_{s}$ & $Q_{s}^{\mathrm{eq}}\left(10^{4}\right)$ & $Q_{r}^{\mathrm{eq}}$ & $\kappa$ & $\tau_{b}$ & $k_{s}$ & $k_{r}$ & $f^{e}$ \\
\hline 10 & 0.1 & $2.5 \times 10^{5}$ & 1.09 & 4.50 & $0.81(2)$ & $1036(22)$ & $1.71 \times 10^{-5}$ & 0.72 & 0.79 \\
\hline 10 & 0.5 & $2.5 \times 10^{5}$ & 1.11 & 4.42 & $0.50(1)$ & $334(5)$ & $5.41 \times 10^{-5}$ & 2.31 & 0.49 \\
\hline 10 & 1 & $2.5 \times 10^{5}$ & 1.12 & 4.44 & $0.33(1)$ & $229(3)$ & $7.67 \times 10^{-5}$ & 3.23 & 0.36 \\
\hline 10 & 5 & $2.5 \times 10^{5}$ & 1.10 & 4.48 & $0.12(1)$ & $142.8(8)$ & $1.27 \times 10^{-4}$ & 5.43 & 0.12 \\
\hline 12 & 0.02 & $6.25 \times 10^{5}$ & 0.169 & 5.03 & $0.92(1)$ & 10296 & $6.44 \times 10^{-7}$ & 0.20 & 0.91 \\
\hline 12 & 0.1 & $4.5 \times 10^{5}$ & 0.164 & 5.16 & $0.72(1)$ & 2549 & $2.61 \times 10^{-6}$ & 0.79 & 0.73 \\
\hline 12 & 1 & $3.0 \times 10^{5}$ & 0.163 & 5.17 & $0.27(1)$ & 702 & $9.46 \times 10^{-6}$ & 2.85 & 0.29 \\
\hline
\end{tabular}

the identical number of scissions and recombinations per unit time and per unit volume can be expressed, respectively, as

$$
\begin{gathered}
n_{s}(\omega)=k_{s}^{0} \phi\left(1-\frac{1}{L_{0}}\right)=\left(2 \omega Q_{s}^{\mathrm{eq}}\right) \phi\left(1-\frac{1}{L_{0}}\right), \\
n_{r}(\omega)=\frac{1}{2} k_{r}^{0}\left(\frac{\phi}{L_{0}}\right)^{2}=\frac{1}{2}\left(2 \omega Q_{r}^{\mathrm{eq}}\right)\left(\frac{\phi}{L_{0}}\right)^{2},
\end{gathered}
$$

where we have expressed the bare rate constants as $k_{s}^{0}$ $=2 \omega Q_{s}^{\mathrm{eq}}$ and $k_{r}^{0}=2 \omega Q_{r}^{\mathrm{eq}}$, introducing $\omega$ independent probabilities $Q_{s}^{\mathrm{eq}}$ and $Q_{r}^{\mathrm{eq}}$ defined below. We still note that the factor 2 in $2 \omega$ expresses the fact that there is a double possibility to trigger a given bonding change through the sampling of one of the two bond connecting arms. For scissions, $Q_{s}^{\text {eq }}$ is the probability that a randomly selected bond is ultimately changed into two chain ends for the MC step under consideration. For recombinations, $Q_{r}^{\mathrm{eq}}$ is the probability that a randomly selected pair of micelles fuse together by connecting any pair of their chain ends into a new bond. Alternatively, in terms of individual arms, $\frac{1}{2} \frac{\phi}{L_{0}} Q_{r}^{\mathrm{eq}}$ is the probability for a randomly selected free arm to form a new bond with any other free arm available in the system.

Regarding $Q_{s}^{\mathrm{eq}} \equiv \int_{\Gamma} d r q_{s}^{\mathrm{eq}}(r)$ and $Q_{r}^{e q} \equiv \int_{\Gamma} d r q_{r}^{\mathrm{eq}}(r)$ as integrals over the distances between involved monomers within the $\Gamma$ region where transitions are allowed, one can express the number of transitions taking place per unit volume and per unit time in a unit range of distances centered on $r$ by

$$
\begin{gathered}
N_{s}(\omega, r)=\left[2 \omega q_{s}^{\mathrm{eq}}(r)\right] \phi\left(1-\frac{1}{L_{0}}\right), \\
N_{r}(\omega, r)=\frac{1}{2}\left[2 \omega q_{r}^{\mathrm{eq}}(r)\right]\left(\frac{\phi}{L_{0}}\right)^{2}
\end{gathered}
$$

for scissions and recombinations, respectively. The microscopic expressions of $q_{s}^{\mathrm{eq}}(r)$ and $q_{r}^{\mathrm{eq}}(r)$ follow now from the MC algorithm detailed in Ref. [26]. Let us define by $P(r)$ the normalized distribution (over the range $[0, R]$ ) of the relative distance between bounded monomers and by $g_{e e}(r)$ the pair distribution function between chain ends (excluding ring closure pairs).

When the sampled arm $i$ is engaged in a bond, the probability for opening this bond within a unit distance interval centered on $r$ is given by

$q_{s}^{\mathrm{eq}}(r)=P(r) P_{s}^{\mathrm{acc}}(r)=P(r)\left\langle\frac{1}{N_{i}+1}\right\rangle \min (1, \exp [-\beta \Delta U(r)])$,

where $P_{s}^{\text {acc }}(r)$, the probability to accept the bond opening, is the product of the usual METROPOLIS acceptance term with $\Delta U(r)=U_{2}(r)-U_{1}(r)$ and an $r$-dependent average (over all bonded arms) of the factor $1 /\left(N_{i}+1\right)$ used in the algorithm to satisfy microscopic reversibility, in connection with the probability to recombine (in the reverse step) the two free arms resulting from the scission under consideration. Specifically, $N_{i}+1$ is the total number of free ends that would be potentially available (in the appropriate $\Gamma$ region) to the originally selected arm $i$ in this reverse case (see Ref. [26]). For the present semidilute solutions, the number of alternative partners $N_{i}$ for such a reverse recombination is found to be zero in most cases so that the average in question is very close to 1.

Our recombination algorithm, which follows the sampling of a free arm in the first place, starts by searching for available free arms at distances within the $\Gamma$ range. If more than one free arm is found, one of them is selected at random and the recombination is then accepted with a probability $P_{r}^{\text {acc }}(r)=\min (1, \exp [+\beta \Delta U(r)])$, where $\Delta U(r)$ was defined previously. Therefore, the probability for the selected arm to find a free arm belonging to another micelle within a unit distance interval centered on $r$ is given by

$$
\begin{aligned}
\frac{1}{2} \frac{\phi}{L_{0}} q_{r}^{\mathrm{eq}} & =4 \pi r^{2} g_{e e}(r) \frac{2 \phi}{L_{0}} P_{r}^{\mathrm{acc}}(r) \\
& =4 \pi r^{2} g_{e e}(r) \frac{2 \phi}{L_{0}} \min (1, \exp [+\beta \Delta U(r)]) .
\end{aligned}
$$

Microscopic reversibility requires that the number of bond breaks and bond recombinations occurring within any 


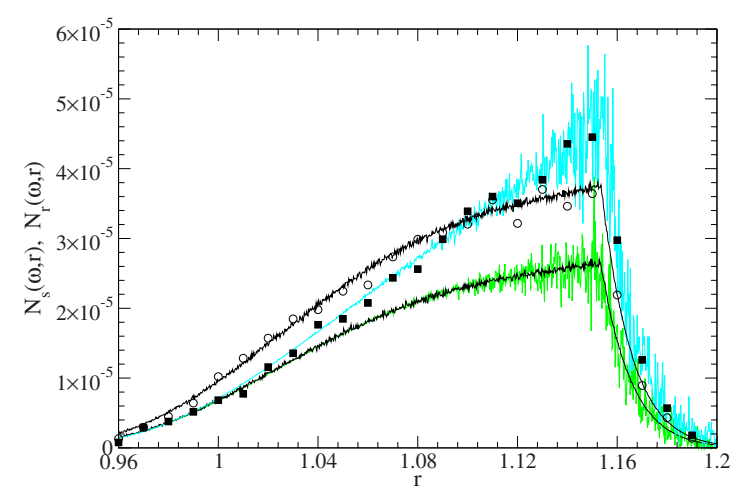

FIG. 2. (Color online) Number of scissions $N_{s}(\omega, r)$ and recombinations $N_{r}(\omega, r)$ per unit volume, unit time, and unit length for the distance $r$ between involved monomers, at equilibrium and under shear flow. The microscopic reversibility is verified at equilibrium, as $N_{s}(\omega, r)$ and $N_{r}(\omega, r)$, shown in green (lower gray) curve and lower black curve, respectively, for the state point $W=10$ with rate $\omega=0.1$, perfectly superimpose. In shear flow, at state point $\dot{\gamma}$ $=0.028, W=10$, and $\omega=0.1$, one observes that the bare rates are increased with respect to results at equilibrium and that $N_{s}(\omega, r)$, shown by the light blue (upper gray) curve, and $N_{r}(\omega, r)$, shown by the upper black curve, are no longer identical (see Sec. V B). For the nonequilibrium case, we also show $N_{s}(\omega, r)$ (filled squares) and $N_{r}(\omega, r)$ (empty circles) obtained by direct counting of scission and recombination events, which coincide with the statistical expressions given by Eqs. (5)-(7).

arbitrary distance interval is equal, thus requiring $N_{s}(\omega, r)$ $=N_{r}(\omega, r)$. We checked this relationship for the state point with $W=10$ for various values of $\omega$ using two equivalent routes.

First, using estimates of $P(r)$ and $g_{e e}(r)$ measured from bead position data extracted from the same simulations, we compute the quantities $N_{s}(\omega, r)$ and $N_{r}(\omega, r)$ using expressions (5)-(7). For $q_{s}$, we did the calculations with the $r$-dependent average $\left\langle 1 /\left(N_{i}+1\right)\right\rangle$ replaced by its average on the full $\Gamma$ interval, neglecting any $r$ coupling for $N_{i}$. Figure 2 shows that the number of scissions and recombinations at a given $r$ is indeed equivalent as it should be, providing a stringent test on the implementation of our kinetic stochastic model.

Alternatively, we did a direct counting of $N_{s}(\omega, r)$ and $N_{r}(\omega, r)$ during the simulations, each accepted scission (recombination) being added in a histogram, normalized in the end by the system volume, by the total length of the simulation and by the bin width. The results (not shown) are in perfect agreement with the curves obtained from structural data shown in Fig. 2.

Furthermore, Fig. 2 shows that $N_{s}(\omega, r)$ [or $\left.N_{r}(\omega, r)\right]$ is getting very small at $r$ values corresponding to the limits of the $\Gamma$ region. This means that, would we have defined the $\Gamma$ region as the full range of the bounded state, namely $[0,1.5]$, the number of transitions would have been only marginally larger by $1 \%$. Indeed, for distances below the lower limit $r$ $=0.96$, the energy gap to go from the (populated) bounded state to the unbounded state is very high (very low acceptance probability), while at distances above $r=1.20$, the same is true for a transition from the (populated) unbounded state toward the bounded state.
The overall probabilities $Q_{s}^{\mathrm{eq}}$ and $Q_{r}^{\mathrm{eq}}$ computed by integration of the $q_{s}^{\mathrm{eq}}$ and $q_{r}^{\mathrm{eq}}$ functions given by Eqs. (6) and (7) are given in Table I. From Table I, we can appreciate how various quantities change, as a result of a change of scission energy from $W$ to $W+\Delta W$. The average length is scaled by a factor $\exp \left(+\beta \frac{\Delta W}{2}\right)$, in agreement with ideal chain standard theory [1] assuming that $\Delta W$ is close to the corresponding scission free-energy difference. Probabilities $Q_{s}^{\mathrm{eq}}$ and $Q_{r}^{\mathrm{eq}}$ are, respectively, scaled to $\approx Q_{s}^{\mathrm{eq}} \exp (-\beta \Delta W)$ for scissions and approximately unchanged for recombination, as can be seen from the integrand functions $q_{s}^{\mathrm{eq}}(r)\left[\right.$ or $\left.q_{r}^{\mathrm{eq}}(r)\right]$ for which the mentioned scaling factor strictly affects the $r$ domain for which $U_{2}(r)<U_{1}(r)$, considering in this analysis that changes in the structural functions $P(r)$ and $g_{e e}(r)$ with $W$ are minor.

\section{B. Effective kinetic constants}

In the explored $\omega$ range, we systematically found in our preliminary studies $[20,26]$ that a significant fraction of scissions are followed by almost immediate recombinations between the same original chain fragments. Such successive accepted "MC moves," taking place within a microscopic time scale without effective change in chain topology, should not contribute to the long-time structural relaxation behavior of the micellar system. This point arises when it is attempted to relate the scission-recombination events observed in our simulations to effective rates, the key parameters $k_{s}$ and $k_{r}$ appearing in the Cates scission-recombination kinetic model which describes the long-time coupling between kinetics and structural relaxation. $k_{s}$ and $k_{r}$ are "effective kinetic rates" which need to be extracted from the long-time behavior of the normalized distribution $\Psi(t)$ of first recombination times. This distribution is accessible in simulations as a histogram of free arm lifetimes $t=t_{2}-t_{1}$, the lifetime being calculated as the elapsed time between the time $t_{1}$ at which a free arm is generated by scission and the time $t_{2}$ it first recombines with another free arm. For pragmatic reasons, $\Psi(t)$ was measured up to a maximum time of $T_{\max }=10^{5}$.

The long-time behavior of $\Psi(t)$ turns out to be exponential and may be written as $\Psi_{\text {asymp }}(t)=\frac{\kappa}{\tau_{b}} \exp \left(-t / \tau_{b}\right)$. The prefactor $\kappa$ is the fraction of effective transitions $[(1-\kappa)$ being the fraction of correlated transitions] while the Poisson distribution involves a time-scale parameter $\tau_{b}$, which represents the effective average lifetime of a free arm or the effective average lifetime of a chain of average length $L_{0}$. The two parameters $\kappa$ and $\tau_{b}$ can be estimated by direct fitting of $\Psi(t)$. But the quoted values in Table I for all investigated cases have been obtained by a cumulative hazard analysis, a more robust method using the same original free arm lifetime data [20]. Figure 3 shows all cases together, plotting $\Psi(t) / \Psi_{\text {asymp }}\left(\tau_{b}\right)$ as a function of $t / \tau_{b}$, in order to show the long-time universal representation of $\Psi(t)$.

Turning now to the short-time behavior, it was found [20] that $\Psi(t)$ is dominated by the contributions from recombinations with the same original partner, and when these contributions are isolated from the recombinations with a new partner, the resulting distribution of self-recombination times follows the power law in $t^{-5 / 4}$ predicted by O'Shaughnessy 


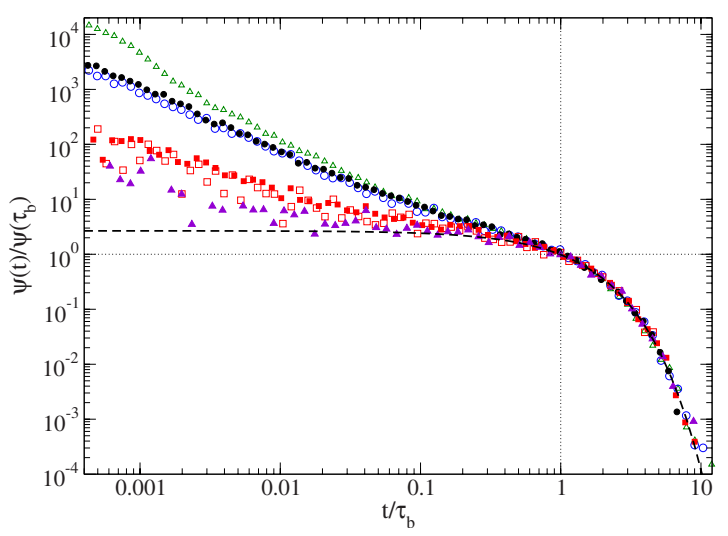

FIG. 3. (Color online) Distribution of first recombinations $\Psi(t)$ for two state points $W=10$ and 12 , for various $\omega$ 's. $\Psi(t)$ is scaled by its mean-field asymptote $\Psi_{\text {asymp }}\left(\tau_{b}\right)$ while $t$ is scaled by $\tau_{b}$. Data are shown for $W=12$ with $\omega=0.02$ (filled triangles), $\omega=0.1$ (filled squares), $\omega=1$ (filled circles); and for $W=10$ with $\omega=0.1$ (open squares), $\omega=1$ (open circles), $\omega=5$ (open triangles). The dashed line is an exponential curve representing the universal long-time behavior.

and $\mathrm{Wu}$ [14]. Data in Table I indicate that for each experiment, the fraction $f^{e}$ of recombinations with a new partner among all recombinations turns out to be systematically close to $\kappa$, providing further evidence of the different nature of transitions responsible for the short- and long-time behavior of $\Psi(t)$, respectively. As the rate $\omega$ decreases, we observe that the fraction of correlated transitions decreases while these transitions occur on a shorter and shorter time scale with respect to the chain end effective lifetime. The $\omega$ $=0.02$ case shows a distribution following the exponential law for $\frac{t}{\tau_{b}} \geqslant 0.02$. We note (figure not shown) that the shorttime behavior of $\Psi(t)$ is identical for the two chain sizes at the same $\omega$ while the fraction of independent recombinations $\kappa$ is slightly lower for the longer chain case (see Table I). This results from the very similar chain end Brownian motion subjected at the same frequency to attempted recombinations whose acceptance probability is essentially 1 at short times and becomes progressively smaller for shorter chains $(W=10)$ as both chain ends drift away and $r$ approaches the upper limit of the $\Gamma$ window.

\section{SCISSION AND RECOMBINATION EVENTS AT EQUILIBRIUM AND TRANSPORT PROPERTIES}

The relaxation dynamics of micelles is expected to be strongly influenced by the scission and recombination kinetics provided the latter is sufficiently fast. We discuss in this section how this point can be made precise for unentangled micellar systems. Previous analyses of the stress relaxation [16] and the monomer diffusion [18] have shown independently that the scission-recombination kinetics leads to the concept of a dynamical segment of size $\Lambda=\left(\tau_{0} k_{s}\right)^{-1 / 3}$, where $\tau_{0}$ is the Rouse microscopic time. The segment size $\Lambda$ is determined by imposing the equality between the segment lifetime and its internal Rouse time, namely $\tau_{\Lambda}=1 /\left(k_{s} \Lambda\right)$ $=\tau_{0} \Lambda^{2}$. The resulting common time
TABLE II. Dynamic segment $\Lambda$, its lifetime $\tau_{\Lambda}$, the MSD crossover time $\tau^{*}$, and the diffusion coefficient $D$ for the cases of $W$ $=10$ and 12 , for various trial frequencies $\omega$.

\begin{tabular}{lllrrc}
\hline \hline$W$ & $\omega$ & \multicolumn{1}{c}{$\Lambda$} & \multicolumn{1}{c}{$\tau_{\Lambda}$} & \multicolumn{1}{c}{$\tau^{*}$} & $D$ \\
\hline 10 & 0.1 & 32.43 & 1788 & 4401 & $2.42 \times 10^{-3}$ \\
10 & 0.5 & 22.02 & 824 & 2283 & $3.14 \times 10^{-3}$ \\
10 & 1 & 19.75 & 663 & 1465 & $3.74 \times 10^{-3}$ \\
10 & 5 & 16.72 & 475 & 1199 & $4.05 \times 10^{-3}$ \\
12 & 0.02 & 97.28 & 16087 & 42076 & $7.01 \times 10^{-4}$ \\
12 & 0.1 & 61.17 & 6361 & 14616 & $1.13 \times 10^{-3}$ \\
12 & 1 & 39.50 & 2653 & 6427 & $1.63 \times 10^{-3}$ \\
\hline \hline
\end{tabular}

$$
\tau_{\Lambda}=\tau_{0}^{1 / 3} k_{s}^{-2 / 3}
$$

is the new structural relaxation time of the micellar system if $\Lambda<L_{0}$. The local dynamics characteristic time value $\tau_{0}$ $=1.7$ was obtained from a preliminary simulation of a monodisperse solution of "dead" polymers (i.e., without scission or recombination) using our micellar model (with $\omega=0$ ) at the same $(\phi, T)$ state point [26]. In the present work, we study two semidilute systems differing only by the average micelle size, and we vary $\omega$ over a large spectrum, ranging from 0.02 to 5 . In Table II, we list the values of $\Lambda$ and $\tau_{\Lambda}$ based on $\tau_{0}$ and the specific rate constant $k_{s}$ obtained previously, confirming that $\omega$ assures $\Lambda<L_{0}$ in all cases. We first focus on the mean-squared displacement (MSD) of monomers, $g(t)$. For a system of unentangled monodisperse dead polymers of length $L$, the MSD evolves from a subdiffusive behavior at short times toward an Einstein diffusion behavior [35], the crossover time $\tau^{*}$ being of the order of the Rouse time $\tau_{R}=\tau_{0} L^{2}$. The behavior of $g(t)$ for micellar solutions is much more complex to predict theoretically, as the monomers belong to a poly-disperse set of chains that continuously break and recombine. All $g(t)$ curves are gathered in Fig. 4 for $\omega=0.1,0.5,1,5$, in the case $W=10$ and for $\omega$

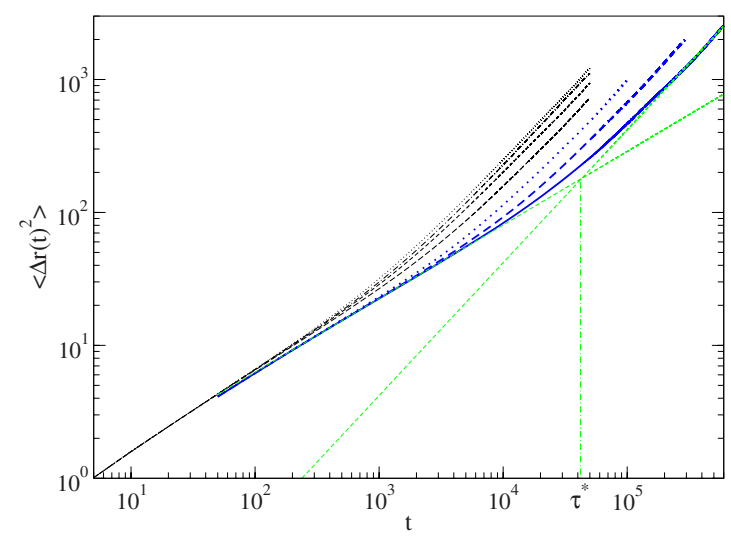

FIG. 4. (Color online) Mean-squared displacement of monomers $g(t)=\left\langle\Delta r(t)^{2}\right\rangle$ for $\omega=5,1,0.5$, and 0.1 of the case $W=10$ and for $\omega=1,0.1$, and 0.02 of the case $W=12$. Data are shown in order from left to right. For $\omega=0.02$, the short-time $\left(t^{0.6}\right)$ and long-time $\left(t^{1}\right)$ asymptotes are drawn (green dashed lines). Their intercept defines the crossover time $\tau^{*}$ (green dashed-dotted line). 


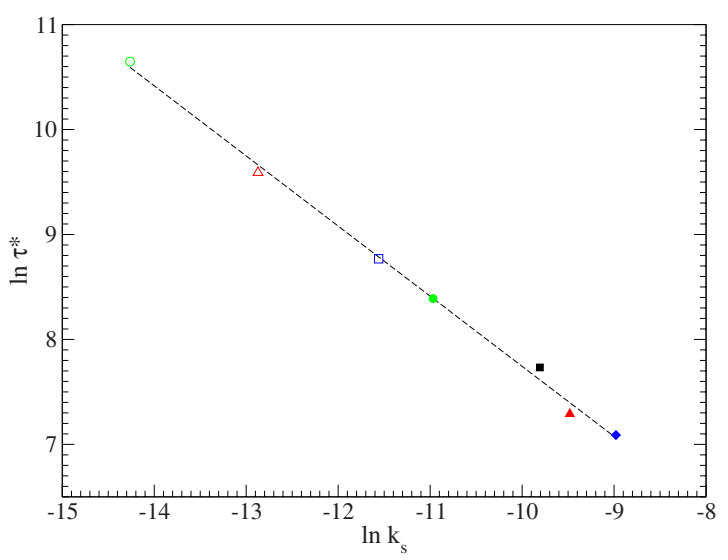

FIG. 5. (Color online) The MSD crossover time $\tau^{*}$, defined in Fig. 4, is plotted vs $k_{s}$ on a log-log scale. Data come from two state points and various $\omega$ values: From left to right, we have, in order, $W=12$ with $\omega=0.02,0.1,1$; and $W=10$ with $\omega=0.1,0.5,1,5$. The dashed line with slope $(-2 / 3)$ corresponds to a power law $\tau^{*}$ $\propto k_{s}^{-2 / 3}$.

$=0.02,0.1,1$ in the case $W=12$. At short times, the MSD is $\omega$ independent and shows a subdiffusive behavior $\left[g(t) \propto t^{0.6}\right]$ while the long-time behavior is diffusive $\left[g(t) \propto t^{1}\right]$. The crossover times $\tau^{*}$, determined on a log-log plot by the intercept between the two asymptotes, are listed in Table II. For a given end-cap energy $W$, the crossover time $\tau^{*}$ diminishes as $\omega$ increases, while for a given $\omega$ value, $\tau^{*}$ increases as $W$ increases. We can expect a link between the monomer dynamics and the kinetics, namely $\tau^{*} \sim \tau_{\Lambda}$, or equivalently $\tau^{*} \sim k_{s}^{-2 / 3}$, using Eq. (8).

In Fig. $5, \tau^{*}$ is plotted versus the corresponding $k_{s}$ value. A two-parameter fitting gives $\ln \tau^{*}=1.06-0.67 \ln k_{s}$, suggesting a power law $\tau^{*} \propto k_{s}^{-2 / 3}$, or equivalently, taking care of prefactors, $\tau^{*} \approx 2.4 \tau_{\Lambda}$, which confirms the relevance of $\tau_{\Lambda}$ as the correct crossover time in the relaxation dynamics of the living polymer chains.

The mean-square displacement in the long-time limit gives the monomer self-diffusion coefficient $D$ (see Table II). For a given $W$ value, $D$ increases with $\omega$, while at fixed $\omega, D$ decreases with $W$. This again can be rationalized in terms of a unique dependency of $D$ in terms of $k_{s}$. As shown in Fig. 6, the diffusion coefficient $D$ scales like $k_{s}^{1 / 3}$ in the explored $\omega$ range, in good agreement with results of the lattice bond fluctuation model (BFM) [18]. This long-time behavior of living polymers can be explained by a diffusion of single monomers within clusters of $\Lambda$ monomers, giving

$$
D \propto \frac{R_{g}^{2}(\Lambda)}{\tau_{\text {Rouse }}(\Lambda)} \propto \frac{\Lambda}{\Lambda^{2}} \propto \Lambda^{-1} \propto k_{s}^{1 / 3},
$$

where $R_{g}(\Lambda)$ denotes the radius of gyration of the cluster. We also note that our results are in agreement with dynamic MC simulations of cylindric micelles based on a slightly different potential model [21].

The way to incorporate the influence of the kinetics on the shear viscosity within unentangled semidilute solutions has been proposed by Faivre and Gardissat [16] within the framework of the Rouse relaxation theory. Taking into ac-

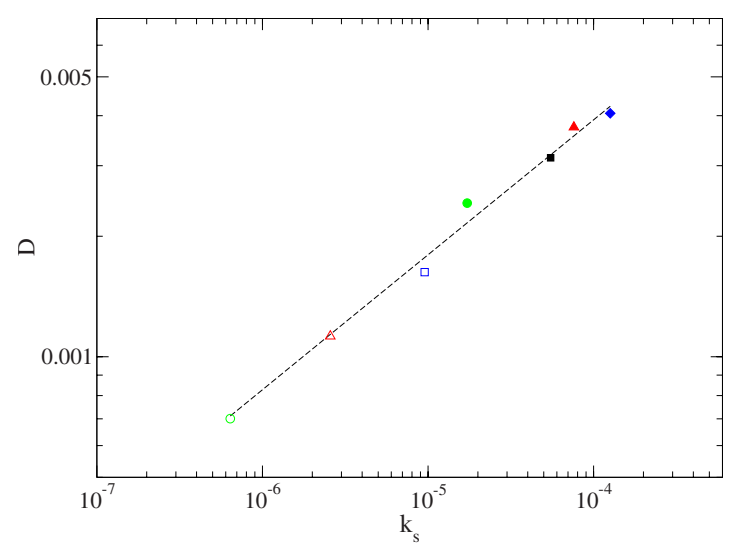

FIG. 6. (Color online) Monomeric Einstein diffusion coefficient as a function of $k_{s}$ for the two state points $(W=10$ and 12). Data are shown in order from left to right for $W=12$ with $\omega=0.02,0.1,1$; and $W=10$ with $\omega=0.1,0.5,1,5$. The value of $D$ is obtained from the long-time behavior of the monomeric mean-square displacement shown in Fig. 4. The dashed line represents a $k_{s}^{1 / 3}$ power law.

count the effects of bond scissions through specific lifetimes for the Rouse modes, they found an explicit expression for the shear modulus relaxation function $G(t)$,

$$
G(t)=\sum_{L=1}^{\infty} W_{L} \frac{G_{0}}{L} \sum_{q=1}^{L} \exp \left\{-\frac{t}{\tau_{\Lambda}}\left[\frac{L}{q \Lambda}+2\left(\frac{q \Lambda}{L}\right)^{2}\right]\right\},
$$

where $W_{L}$ is the equilibrium micelle length distribution, $\Lambda$ and $\tau_{\Lambda}$ are defined previously in this section, and $G_{0}$ is the elastic modulus. For $L_{0} \gg \Lambda$ and $t>\tau_{\Lambda}$, Eq. (10) becomes

$$
G(t)=\left(\frac{\pi}{6}\right)^{1 / 2} G_{0}\left(\frac{\tau_{0}}{t}\right)^{1 / 2} \exp \left(-\frac{3}{2^{1 / 3}} \frac{t}{\tau_{\Lambda}}\right) .
$$

The corresponding zero-shear viscosity, obtained by integrating Eq. (11), gives

$$
\eta_{0}=\left(\frac{\pi}{3}\right)^{1 / 2} G_{0} \tau_{0}^{1 / 2} 2^{-1 / 3} \tau_{\Lambda}^{1 / 2}=\left(\frac{\pi}{3}\right)^{1 / 2} G_{0} 2^{-1 / 3} \tau_{0}^{2 / 3} k_{s}^{-1 / 3}
$$

It is interesting to observe that, for unentangled micelle solutions, the general dependency of the static viscosity on the relevant physical parameters $(\phi, T, \omega, W)$ can be reexpressed as an explicit function of $\phi, T, k_{s}$, and $L_{0}$. In the regime where $\Lambda \approx L_{0}$, we expect $\eta_{0}=\eta_{0}\left(\phi, T, k_{s}, L_{0}\right)$, whereas for systems with $\Lambda \gg L_{0}$ (very slow kinetics) we expect $\eta_{0}=\eta_{0}\left(\phi, T, L_{0}\right)$ (independent of $\Lambda$ ), as the relaxation would be purely Rouse-like. In the conditions of our simulations where $\Lambda<L_{0}$, Eq. (12) predicts that the $L_{0}$ dependency drops out, and $\eta_{0}$ is a simple power law of $k_{s}$ or $\tau_{\Lambda}$, with $G_{0}$ depending on $\phi$ and $T\left(G_{0}=\phi k_{B} T\right)$.

The expression $\eta_{0} \propto k_{s}^{-1 / 3}$ is tested here using our zeroshear viscosity data, which come from our two-state points and various $\omega$ 's. These viscosity values were not estimated from dynamical fluctuations as earlier [20,22], but by a more precise numerical procedure based on an extrapolation of nonequilibrium simulation results obtained in experiments 


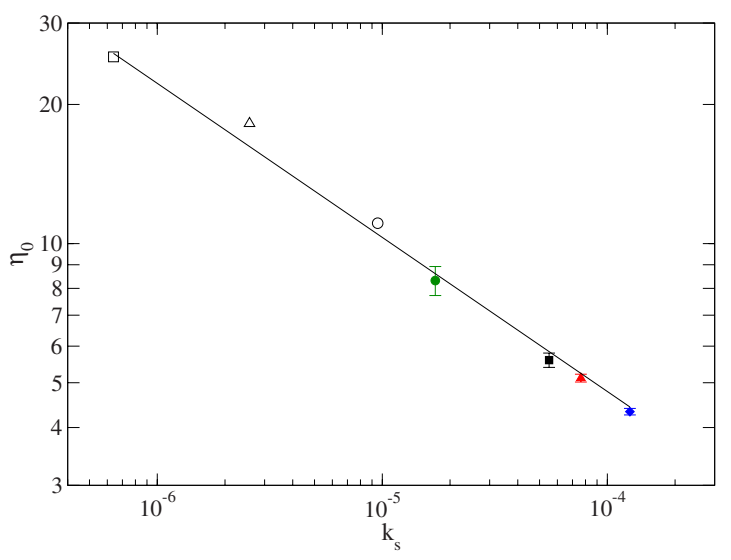

FIG. 7. (Color online) Zero-shear viscosity $\eta_{0}$, as obtained by extrapolation of nonequilibrium data (see text) vs the effective rate constant $k_{s}$ measured as explained in Sec. III for two state points and various $\omega$ 's. Data are shown in order from left to right for the state points of $W=12$ with $\omega=0.02,0.1,1$; and $W=10$ with $\omega$ $=0.1,0.5,1,5$. The straight line is the theoretical prediction Eq. (12) of Faivre and Gardissat.

where our system is put under stationary shear flow. The procedure was briefly described in Ref. [19], and will be explained in more detail in the next section. All thus obtained values of $\eta_{0}$ are shown in Fig. 7 as a function of $k_{s}$. Our data follow very well the $k_{s}^{-1 / 3}$ law, independently of $W$. The best fit of these data by the Faivre-Gardissat Eq. (12), using $G_{0}$ as the only adjustable parameter, gives $G_{0}=0.19$, which is quite close to our previous estimate of $G_{0}=0.26$ based on quite noisy equilibrium $G(t)$ (see Ref. [20]). Notice also that our estimate is very close to the one given by the theoretical expression $G_{0}=\phi k_{B} T=0.15$, the excess part being attributed to short-time behavior of $G(t)$ not taken into account in Eq. (12). This confirms once more the theory of Faivre and Gardissat, valid for fast kinetics.

\section{NONEQUILIBRIUM PROPERTIES}

In this part we are interested in the rheology and the kinetics of our system under stationary shear flow. Nonequilibrium simulations, using the technique exposed in Sec. II, are performed on systems with a given set of parameters $\left(L_{0}, \omega, \dot{\gamma}\right)$. The results come from the same two-state points as in equilibrium, with, respectively, $L_{0}=56$ and 151 . The trial frequency $\omega$ takes, for each state point, the same values as indicated in Table I. The shear rate values considered are in the range $5.6 \times 10^{-5}<\dot{\gamma}<0.21$, with, for each couple $\left(L_{0}, \omega\right)$, a specific range such that the reduced rate $\beta_{\Lambda}=\dot{\gamma} \tau_{\Lambda}$ lies in the range $0.1<\beta_{\Lambda} \leq 100$. At the setting up of the flow, the system is equilibrated for a transient period of $T_{\text {trans }}=2$ $\times 10^{4}$, then statistics are collected for runs of typically $T_{\text {stat }}$ $=2.5 \times 10^{5}$.

The first part of this section deals with the collective rheological behavior. The second part analyzes the kinetics under flow.

\section{A. Collective material functions}

Under shear flow, chains are oriented and deformed. Typically, these phenomena are studied experimentally by bire- fringence and neutron or light scatterings. They can be measured through the anisotropy arising in tensorial quantities such as the order-parameter tensor $\overline{\overline{\mathbf{O}}}$ and the gyration tensor $\overline{\mathbf{G}}$. The former is given by

$$
\overline{\overline{\mathbf{O}}}=\frac{1}{N_{b}} \sum_{i=1}^{N_{b}}\left\langle\frac{3}{2} \frac{\mathbf{u}_{i} \mathbf{u}_{i}}{|\mathbf{u}|^{2}}-\frac{\overline{\overline{\mathbf{I}}}}{2}\right\rangle,
$$

where $\mathbf{u}_{i}$ is the unit vector joining two adjacent monomers, $\overline{\overline{\mathbf{I}}}$ is the unit tensor, $N_{b}$ is the total number of bonds, and $\langle\cdots\rangle$ denotes the statistical average. For a given micelle length $L$, we can define the gyration tensor as follows:

$$
\overline{\overline{\mathbf{G}}}(L)=\frac{1}{2 L^{2}} \sum_{i, j=1}^{L}\left\langle\left(\mathbf{R}_{i}-\mathbf{R}_{j}\right)\left(\mathbf{R}_{i}-\mathbf{R}_{j}\right)\right\rangle,
$$

where $\mathbf{R}_{i}$ are the coordinates of the $i$ th monomer in the chain. In a polydisperse micellar solution, one can measure the $z$-averaged elements [33],

$$
\overline{\overline{\mathbf{G}}}=\int d L L^{2} P(L) \overline{\overline{\mathbf{G}}}(L) / \int d L L^{2} P(L),
$$

where $P(L)$ is the distribution of micelle lengths. The mechanic response of the system is conventionally measured by the stress tensor $\overline{\bar{\sigma}}$. Its microscopic expression, for the $\alpha \beta$ component, is given by

$$
\sigma_{\alpha \beta}=-\frac{1}{V}\left\langle\left(\sum_{i=1}^{M} m v_{i \alpha} v_{i \beta}+\sum_{i=1}^{M-1} \sum_{j=i+1}^{M} r_{i j \alpha} F_{i j \beta}\right)\right\rangle,
$$

where $v_{i \alpha}$ is the $\alpha$ component of the velocity of particle $i, r_{i j \alpha}$ is the $\alpha$ component of the vector from the position of particle $j$ to particle $i, F_{i j \beta}$ is the $\beta$ component of the force exerted by particle $j$ on particle $i$, and $M$ is the total number of monomers in the system.

At equilibrium, the system is isotropic and the tensorial quantity, say $\overline{\overline{\mathbf{A}}}$, reduces to a scalar quantity, multiplied by $\overline{\overline{\mathbf{I}}}$. With the shear flow along the $x$ axis and gradient along the $y$ axis, the angle $\chi_{A}$, defined through the relation

$$
\cot \left(2 \chi_{A}\right)=\frac{A_{x x}-A_{y y}}{2 A_{x y}},
$$

measures the rotation angle around the $z$ axis in order to bring the tensor $\overline{\overline{\mathbf{A}}}$ in its principal axes $(I, I I, I I I)$. As the shear rate $\dot{\gamma}$ increases from zero, $A_{x y}$ starts linearly with $\dot{\gamma}$, while the first contribution to $A_{x x}-A_{y y}$ is of order $\dot{\gamma}^{2}$ (see Ref. [35]). Therefore, the linear (Newtonian) regime is characterized by $\chi_{A}=\pi / 4$. Outside the linear regime $\chi_{A}$ decreases to zero for increasing shear rates $[36,37]$. The extinction angle $\chi_{O}$ measures the average orientation of the bonds. We have computed this quantity according to Eqs. (13) and (17). Figure 8 shows $\chi_{O}$ versus the reduced shear rate $\beta_{\Lambda}$. This angle decreases from $45^{\circ}$ at very low shear rate down to a few degrees while $\beta_{\Lambda}=100$. All the data follow a universal curve, $\chi_{O}\left(\beta_{\Lambda}\right)$, which can be approximated by an interpolation formula of the kind suggested in [37]: $\chi_{O}\left(\beta_{\Lambda}\right)$ $=\chi_{O}(0)\left(1+\beta_{\Lambda}\right)^{-0.51}$. We notice some discrepancy between our results and the fit at very high shear rates $\beta_{\Lambda}>20$, which 


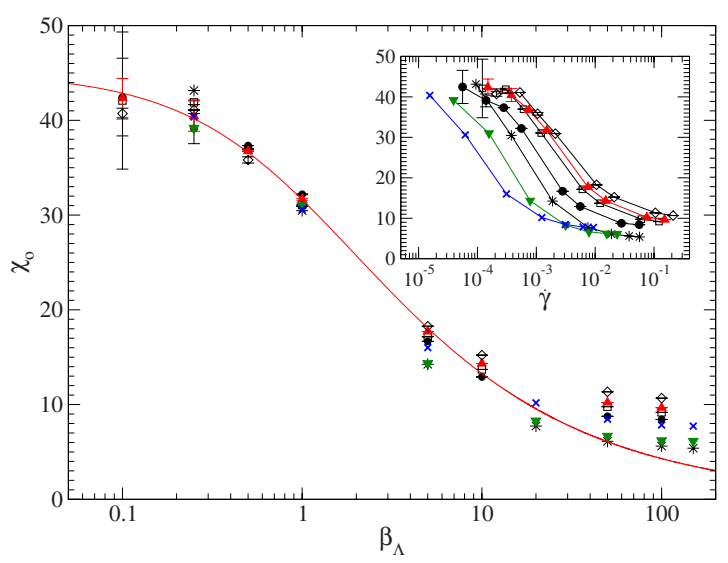

FIG. 8. (Color online) The extinction angle $\chi_{O}$ vs $\beta_{\Lambda}$ and vs $\dot{\gamma}$ (inset), for both semidilute cases. Data correspond to $W=12$, with $\omega=0.02$ (cross), $\omega=0.1$ (down triangles), $\omega=1$ (stars); and $W=10$ with $\omega=0.1$ (filled circles), $\omega=0.5$ (open squares), $\omega=1$ (up triangles), $\omega=5$ (open diamonds). The continuous line in the main figure corresponds to the empirical law (see text). In the inset, lines connecting the data points are a guide to the eye.

could be originating from finite-size effects in strongly shear cases. For comparison, the inset of Fig. 8 shows a dispersion of the $\chi_{O}$ functions when the bare shear rate $\dot{\gamma}$ is used as an independent variable. As expected, one sees that for fixed $L_{0}$ and $\dot{\gamma}$, the angle $\chi_{O}$ is larger when $\omega$ increases, reflecting the fact that the average alignment of the bonds is weaker when the scission-recombinations are faster.

In Fig. 9, we show, for selected parameter sets, the orientation angle of the stress tensor $\chi_{\sigma}$ versus $\beta_{\Lambda}$. For the sake of comparison, the angle $\chi_{O}$ versus $\beta_{\Lambda}$ and the empirical law $\chi_{O}\left(\beta_{\Lambda}\right)$ are also plotted. This figure indicates that for a given $\beta_{\Lambda}$, the angles $\chi_{O}$ and $\chi_{\sigma}$ are very nearly identical. Such a result is a confirmation of the well known stress-optical law [35], even in quite nonlinear regime $\beta_{\Lambda} \gg 1$.

The size of the chains is typically measured by the gyration tensor, which is diagonal at equilibrium, showing the isotropic nature of the chains. Under shear flow, the anisotropy of the chains can be evidenced by the orientational

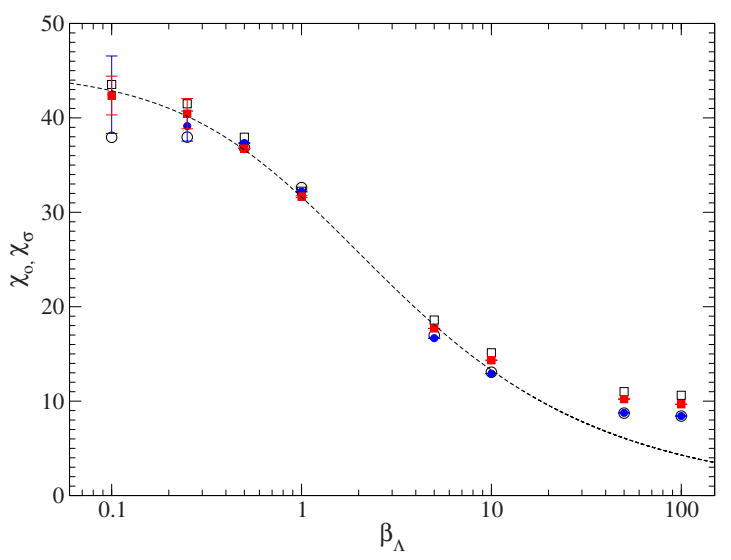

FIG. 9. (Color online) $\chi_{\sigma}$ (angle of stress tensor) vs $\beta_{\Lambda}$, and $\chi_{O}$ vs $\beta_{\Lambda}$, for the state point of $W=10$ with $\omega=0.1$ (circles) and $\omega=1$ (squares) (filled symbols represent $\chi_{O}$ and open symbols represent $\left.\chi_{\sigma}\right)$. The dashed line shows the empirical law for $\chi_{O}$ (see text).

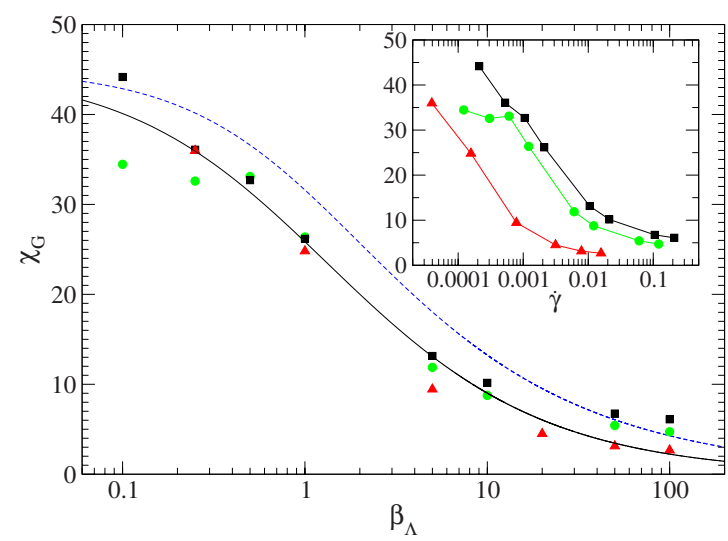

FIG. 10. (Color online) $\chi_{G}$ (the orientation angle associated with the average gyration tensor) vs $\beta_{\Lambda}$, and vs $\dot{\gamma}$ (inset), for the two state points and several $\omega$ 's. Data are shown for $W=12$ with $\omega$ $=0.1$ (triangles) and $W=10$ with $\omega=0.5$ (dots), $\omega=5$ (squares). The continuous line in the main figure corresponds to the fit to $\chi_{G}$ (see text) and the dashed line is the universal curve of $\chi_{O}$, already shown in Fig. 9. In the inset, lines connecting the data points are a guide to the eye.

angle $\chi_{G}$, the angle of the principal axis I of the averaged gyration tensor $\overline{\overline{\mathbf{G}}}$ with respect to the flow direction. Not surprisingly, this function is again a universal function of the reduced shear rate $\beta_{\Lambda}$. In Fig. 10, we show $\chi_{G}$ versus $\beta_{\Lambda}$ and $\chi_{G}$ versus $\dot{\gamma}$ (inset). A numerical fit was made for the data $\chi_{G}\left(\beta_{\Lambda}\right)$, which seems to be most valid for $0.2<\beta_{\Lambda}<20$. It gives $\chi_{G}\left(\beta_{\Lambda}\right)=\chi_{G}(0)\left(1+\beta_{\Lambda}^{0.81}\right)^{-0.80}$. We see that $\chi_{G}$ lies systematically below $\chi_{O}$, as observed for monodisperse polymers $[36,37]$. This result reflects the fact that the deformation of whole micelles is more sensitive to the flow than the orientation of the individual bonds.

The degree of orientation of the bonds with respect to the flow direction can be described by the orientation order parameter $[38,39]$. We compute $S_{I}$, the largest eigenvalue of $\overline{\mathbf{O}}$, for various sets of parameters. $S_{I}$ evolves monotonically from 0 (no shear) to 1 (high shear). We showed previously in a preliminary study [19] that for a given $L_{0}, S_{I}$ behaves as a universal function of $\beta_{\Lambda}$ for different $\omega$ 's. Remarkably, this dependency remains valid for the longer chain system [40] (i.e., $L_{0}=151$ ). Another interest of $S_{I}$ comes from its link with the rheology. A recent experimental work on micellar solutions [38] showed that the non-Newtonian viscosity $\eta$ is a simple decreasing exponential function of $S_{I}$, and we have tested this observation in our system. In our nonequilibrium simulations, the average stress tensor is estimated by Eq. (16). Under a shear flow in the $x$ direction, the effective viscosity $\eta(\omega, \dot{\gamma})$ is obtained by the following:

$$
\eta(\omega, \dot{\gamma})=\frac{\sigma_{x y}(\omega, \dot{\gamma})}{\dot{\gamma}} .
$$

All our results can be fitted by a set of single exponential laws $\eta=\eta_{0}(\omega) \exp \left(-a S_{I}\right)$, where $\eta_{0}(\omega)$ is the zero-shear viscosity and $a=5.7$ for all $\omega$ and both state points. Thus, as mentioned in the previous section, our nonequilibrium data give, by extrapolation, an accurate estimation of the zero- 


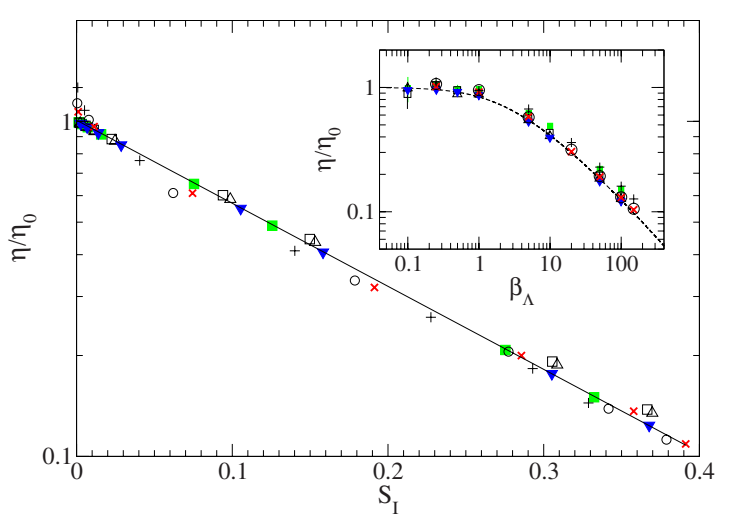

FIG. 11. (Color online) Universal function $\eta / \eta_{0}$ vs $S_{I}$. Results for the two state points and all $\omega$ 's are shown: $W=12$ with $\omega$ $=0.02(+$ symbols $), \omega=0.1$ (circles), $\omega=1$ (cross); and $W=10$ with $\omega=0.1$ (filled squares), $\omega=0.5$ (open squares), $\omega=1$ (open triangles), $\omega=5$ (filled down-triangles). The straight line indicates the exponential law. In the inset, the universal function $\eta / \eta_{0}$ vs $\beta_{\Lambda}$ is drawn (dashed line).

shear viscosity $\eta_{0}(W, \omega)$, for given $T$ and $\phi$. These observations also lead to shear thinning parameter $\eta / \eta_{0}$ being a universal function of the order parameter $S_{I}$, as shown in Fig. 11. In the inset, we plot $\eta / \eta_{0}$ as a function of $\beta_{\Lambda}$. We observe that this quantity falls on a universal curve, which obeys $\eta / \eta_{0}=\left(1+\beta_{\Lambda}\right)^{-0.37}$, as in our previous work [19] based on a single state point $(W=10)$.

\section{B. Scission-recombination kinetics under flow}

In this subsection, the influence of the shear flow on the kinetics is studied. In Sec. II, we stressed that our kinetics model, coupled to a Langevin dynamics for monomer diffusion, guarantees that the number of scissions and recombinations (per unit volume and unit time) taking place at any given relative distance $r$ between monomers is equal at equilibrium.

Under shear, as a result of the imposition of a shear flow in the Langevin dynamics, the microscopic reversibility is broken [41]. Figure 2 shows for a specific nonequilibrium stationary state the $r$-dependent number of scissions and recombinations $N_{s}^{\text {neq }}(r)$ and $N_{r}^{\text {neq }}(r)$ (where the dependency on $\omega$ and $\dot{\gamma}$ is kept implicit), which are natural extensions of similar quantities defined at equilibrium in Eq. (5). We have computed $N_{s}^{\text {neq }}(r)$ and $N_{r}^{\text {neq }}(r)$ according to the right-hand side of Eq. (5) using expressions (6) and (7) in which the bond length distribution $P(r)$ and the chain end distribution function $g_{e e}(r)$ were computed from data provided by our simulation of the (sheared) nonequilibrium stationary state. The transitions were also computed by direct counting of events as a function of $r$ and the results are in very good agreement with the prediction based on structural data. We stress that Fig. 2 shows that the modification on the kinetics due to shear flow takes place essentially within the adopted $\Gamma$ range. Indeed, as in equilibrium, an extension of the $\Gamma$ range by lowering its lower limit or increasing its upper limit would have a marginal impact on our results and conclu- sions, as only a few additional scission or recombination events of the order of $1 \%$ would be allowed.

The analysis of nonequilibrium data in Fig. 2 leads to two important remarks.

Under shear flow, scission events occur more often than recombinations at large distances while recombinations occur more often than scissions at short distances. This does not contradict the fact that the total number of scissions and recombinations [corresponding to the integrals of $N_{s}(\omega, r)$ and $\left.N_{r}(\omega, r)\right]$ maintains the equality $n_{s}=n_{r}$ required for a stationary state. The time independence of the distributions $P(r)$ and $g_{e e}(r)$ under stationary shear flow thus implies that there is, on average, an overall continuous extension of bounded pairs and an overall continuous contraction of the relative distances between chain ends pertaining to different micelles.

The comparison of the equilibrium and nonequilibrium results shown in Fig. 2, which only differ by the imposition or not of a shear rate, shows an enhancement of the number of transitions in shear flow with respect to equilibrium. This can be rationalized as follows. As the shear flow induces on average an elongation of the bounded pairs, that is, a shift of $P(r)$ toward larger $r$ values, the Monte Carlo scission acceptance probability is enhanced by the corresponding systematic decrease of the energy gap between the bounded to the unbounded state as $r$ increases. As no corresponding shear effect takes place on the chain end distribution function, the only way for the system to readjust global equivalence between both types of transitions is to increase the density of chain ends, namely decreasing the average size of the chains under shear with respect to equilibrium. The shortening of the micelles under shear, which has been found quite systematically in previous studies where a continuous potential was used to represent the scission and recombination processes $[17,24,25]$, is analyzed in further detail below.

The rate constants can be computed, under stationary flow, by the cumulative hazard method. Results are obtained for one state point $(W=10)$ and various $\omega$. We plot $k_{s}$ and $k_{r}$, scaled by their equilibrium values, versus the bare shear rate $\dot{\gamma}$, in Fig. 12. We see that $k_{s}$ increases much more rapidly with $\dot{\gamma}$ than $k_{r}$, showing the effect of the stretching of chains. Secondly, results for different $\omega$ fall on one universal curve. A good fit gives

$$
k_{s}(\omega, \dot{\gamma})=2 \omega \kappa(\omega, \dot{\gamma}) Q_{s}(\omega, \dot{\gamma})=2 \omega \kappa_{0}(\omega) Q_{s}^{\mathrm{eq}}(1+18.5|\dot{\gamma}|),
$$

$$
k_{r}(\omega, \dot{\gamma})=2 \omega \kappa(\omega, \dot{\gamma}) Q_{r}(\omega, \dot{\gamma})=2 \omega \kappa_{0}(\omega) Q_{r}^{\mathrm{eq}}(1+2.5|\dot{\gamma}|) .
$$

Equations (19) and (20) show a separation of the dependencies on $\omega$ and on $\dot{\gamma}$, the $\omega$ dependence being included in the (static) transmission coefficient $\kappa_{0}(\omega)$ and the shear flow influence by the $\dot{\gamma}$-dependent term.

These results on rate constants indicate how the average length $\langle L\rangle$ decreases with increasing shear rate, and can explain why it varies independently from $\omega$, as in the equilibrium case $[20,19]$. This independence of $\langle L\rangle$ on $\omega$ under shear flow differs from the other collective properties exam- 


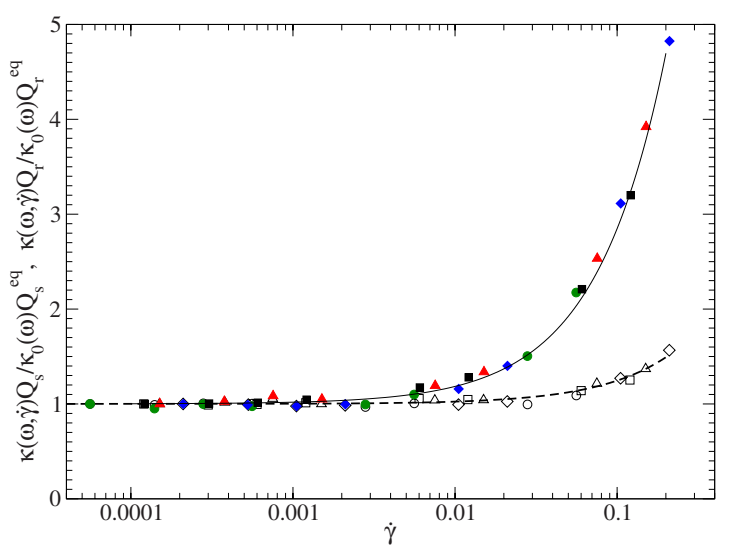

FIG. 12. (Color online) Kinetic rate constants reduced by their equilibrium values vs the shear rate $\dot{\gamma}$, for the case of $W=10$ with $\omega=0.1$ (circles), $\omega=0.5$ (squares), $\omega=1$ (triangles), and $\omega=5$ (diamonds). The open symbols represent the data of $\frac{\kappa(\omega, \dot{\gamma}) Q_{r}}{\kappa_{0}(\omega) Q_{r}^{\text {eq }}}$ and the filled symbols represent the data of $\frac{\kappa(\omega, \dot{\gamma}) Q_{s}}{\kappa_{0}(\omega) Q_{s}^{\text {eq }}}$. The continuous and dashed lines correspond to the fit to the data (see text).

ined above, which yield universal functions of the reduced shear rate $\beta_{\Lambda}$. The equality between effective scissions and effective recombinations requires

$$
\begin{aligned}
n_{s}^{\mathrm{eff}}(\omega, \dot{\gamma}) & =k_{s}(\omega, \dot{\gamma}) \phi(1-1 /\langle L\rangle) \\
& =n_{r}^{\mathrm{eff}}(\omega, \dot{\gamma})=\frac{1}{2}\left(\frac{\phi}{\langle L\rangle}\right)^{2} k_{r}(\omega, \dot{\gamma})
\end{aligned}
$$

This gives, taking into account Eqs. (19) and (20) and assuming $\langle L\rangle \gg 1$,

$$
\langle L\rangle=\sqrt{k_{r} \phi / 2 k_{s}}=L_{0} \sqrt{\frac{1+2.5|\dot{\gamma}|}{1+18.5|\dot{\gamma}|}} .
$$

In Fig. 13, the evolution of $L_{0} /\langle L\rangle$ is shown versus $\dot{\gamma}$, for four values of $\omega$ and the state point $W=10$. All points lay on the same curve, given by Eq. (22).

\section{CONCLUSIONS}

This work concerns micellar solutions in a semidilute regime where chains are dynamically unentangled. Using a recent "equilibrium polymer" model, we studied two state points $(W, \phi)$ corresponding to chains with different average size. Given our ability to tune (through the adjustment of the $\omega$ parameter) the barrier height of the kinetic processes without modifying the structural and thermodynamic properties of the system, we have followed the influence of the scission-recombination kinetics on various relaxation processes at equilibrium and on the stress and structural features when these solutions are sheared. Our scissionrecombination kinetic model is investigated in some detail at equilibrium (where microscopic reversibility is verified) and

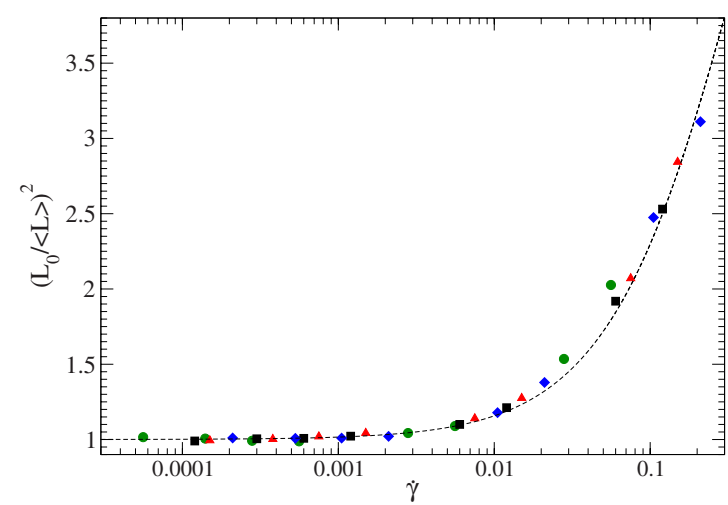

FIG. 13. (Color online) Evolution of $\left(L_{0} /\langle L\rangle\right)^{2}$ as a function of the shear rate $\dot{\gamma}$ for the case of $W=10$ with $\omega=0.1$ (circles), $\omega$ $=0.5$ (squares), $\omega=1$ (triangles), and $\omega=5$ (diamonds). The dashed line corresponds to the function $\left(\frac{L_{0}}{\langle L\rangle}\right)^{2}=\frac{1+18.5|\dot{\gamma}|}{1+2.5|\dot{\gamma}|}$ (see text).

under flowing conditions, given its intrinsic interest to model the kinetics of various adsorption or reaction processes in complex systems modeled at a mesoscopic scale. The present study contrasts with previous simulation studies $[17,24,25,42]$ by the capability of our model to follow, for each thermodynamic system, not only the shear rate $\dot{\gamma}$ dependency as usual, but also the dependency of various structural or dynamical properties with the size $\Lambda$ of its dynamical units, a crucial parameter directly linked to the rates of the scission-recombination kinetics. At equilibrium, the relevant time $\tau_{\Lambda} \propto k_{s}^{-2 / 3}$ appears to be the crossover time between Rouse and Einstein diffusive regimes and is shown to be the characteristic time relevant to the Faivre-Gardissat theoretical expression of the Newtonian shear viscosity expression for unentangled polymers subject to scission-recombination kinetics. We show more evidence of the universal role of the dynamic time $\tau_{\Lambda}$ by displaying that the chain orientation, chain deformation, and the shear thinning behavior are all universal functions of the reduced shear rate $\beta_{\Lambda}=\tau_{\Lambda} \dot{\gamma}$. For future work, we plan to investigate dynamically entangled micelles. An interesting regime corresponds to the situation in which the kinetics is fast compared to the Rouse time of the average chain segment between two successive entanglements $[43,44]$, which should give rise to a specific stress relaxation mechanism and associated relaxation time suggested in [13].

\section{ACKNOWLEDGMENTS}

This work has benefitted from numerous discussions with J. Wittmer of the Ch. Sadron Institute and with M. Baus of the Univeristé Libre de Bruxelles. Thanks are also due to $\mathrm{G}$. Destrée of the Centre de Calcul of the ULB/VUB for technical help. C.C.H. was financially supported partly by the Action de Recherche Concertée No. 00/05-257 of the Communauté Française de Belgique and partly by the Région Lorraine (France). The computing resources of the French center CINES and of the PMMS of the Universite de Metz are gratefully acknowledged. 
[1] M. E. Cates and S. J. Candau, J. Phys.: Condens. Matter 2, 6869 (1990).

[2] P. van der Schoot, in Supramolecular polymers, edited by A. Ciferri (New York, 2005), p. 77.

[3] M. E. Cates and S. M. Fielding, Adv. Phys. 55, 799 (2006).

[4] Giant micelles: Properties and applications, edited by R. Zana and E. W. Kaler (Taylor \& Francis, New York, 2007).

[5] C. A. Dreiss, Soft Matter 3, 956 (2007).

[6] G. C. Maitland, Curr. Opin. Colloid Interface Sci. 5, 301 (2000).

[7] S. Lerouge, J. P. Decruppe, and C. Humbert, Phys. Rev. Lett. 81, 5457 (1998).

[8] H. Rehage and H. Hoffmann, Rheol. Acta 21, 561 (1982).

[9] N. A. Spenley, M. E. Cates, and T. C. B. McLeish, Phys. Rev. Lett. 71, 939 (1993).

[10] S. M. Fielding and P. D. Olmsted, Phys. Rev. Lett. 90, 224501 (2003).

[11] M. E. Cates, Macromolecules 20, 2289 (1987).

[12] P. G. de Gennes, Scaling Concepts in Polymer Physics (Cornell University Press, Ithaca, NY, 1979).

[13] M. E. Cates, Europhys. Lett. 4, 497 (1987).

[14] B. O’Shaughnessy and J. Yu, Phys. Rev. Lett. 74, 4329 (1995).

[15] I. A. Nyrkova and A. N. Semenov, Europhys. Lett. 79, 66007 (2007).

[16] G. Faivre and J. L. Gardissat, Macromolecules 19, 1988 (1986).

[17] M. Kroger and R. Makhloufi, Phys. Rev. E 53, 2531 (1996).

[18] A. Milchev, in Computational Methods in Colloid and Interface Science, edited by M. Borowko (Dekker, New York, 2000), p. 510.

[19] C. C. Huang, H. Xu, and J. P. Ryckaert, Europhys. Lett. 81, 58002 (2008).

[20] C. C. Huang, H. Xu, and J. P. Ryckaert, J. Chem. Phys. 125, 094901 (2006); 128, 069901 (2008).

[21] F. Crevel, Ph.D. thesis, University Louis Pasteur Strasbourg (2007).

[22] J. T. Padding and E. S. Boek, Europhys. Lett. 66, 756 (2004).

[23] E. S. Boek, W. K. den Otter, W. J. Briels, and D. Iakovlev, Philos. Trans. R. Soc. London, Ser. A 362, 1625 (2004).

[24] A. Milchev, J. P. Wittmer, and D. P. Landau, Eur. Phys. J. B
12, 241 (1999).

[25] J. T. Padding, E. S. Boek, and W. J. Briels, J. Chem. Phys. 129, 074903 (2008).

[26] C. C. Huang, H. Xu, J. P. Wittmer, F. Crevel, and J. P. Ryckaert, Lecture Notes in Physics Vol. 704, edited by Ferrario, Binder, and Ciccotti (Springer-Verlag, Berlin, 2006), p. 379.

[27] K. Kremer and G. S. Grest, J. Chem. Phys. 92, 5057 (1990).

[28] M. P. Allen and D. J. Tildesley, Computer Simulation of Liquids (Clarendon, Oxford, 1987).

[29] A. V. Lyulin, D. B. Adolf, and G. R. Davies, J. Chem. Phys. 111, 758 (1999).

[30] C. Pierleoni and J. P. Ryckaert, J. Chem. Phys. 113, 5545 (2000).

[31] J. P. Hansen and I. R. McDonald, Theory of Simple Liquids, 2nd ed. (Academic, London, 1986).

[32] M. G. Paterlini and D. M. Ferguson, Chem. Phys. 236, 243 (1998).

[33] M. Rubinstein and R. H. Colby, Polymer Physics (Oxford University Press, Oxford, 2003).

[34] R. Everaers et al., Science 303, 823 (2004).

[35] M. Doi and S. F. Edwards, The Theory of Polymer Dynamics (Clarendon, Oxford, 1986).

[36] C. Pierleoni and J. P. Ryckaert, Macromolecules 28, 5097 (1995).

[37] C. Aust, M. Kröger, and S. Hess, Macromolecules 32, 5660 (1999).

[38] S. Förster, M. Konrad, and P. Lindner, Phys. Rev. Lett. 94, 017803 (2005).

[39] V. Croce, T. Cosgrove, C. Dreiss, S. King, G. Maitland, and T. Hughes, Langmuir 21, 6762 (2005).

[40] C. C. Huang, Ph.D. thesis, University Paul Verlaine-Metz (2007).

[41] U. Seifert, in Soft Matter, Synthetic to Biological Materials, Lecture Notes of the 39th IFF Spring School, edited by J. Dhont et al. (2008).

[42] J. T. Padding and E. S. Boek, Phys. Rev. E 70, 031502 (2004).

[43] A. Khatory, F. Lequeux, F. Kern, and S. J. Candau, Langmuir 9, 1456 (1993).

[44] S. R. Raghavan and E. W. Kaler, Langmuir 17, 300 (2001). 